participants and $32 \%$ of the non-EUS participants were alive. EUS improved survival adjusted for generic quality of life with an HR of 0.69 (95\% CI 0.49 to 0.98 ); and both its components-survival and EQ5D scores; the benefits of EUS were significantly greater for those with poor initial quality of life, but there was no difference between centres. Similarly, there was a significant interaction between initial quality of life and the effect of EUS on all the FACT scales; again sicker patients benefitted more from EUS. However, there was no significant difference between EUS and non-EUS groups in mean FACT scores adjusted for covariates. Both management plans and final treatment varied between centres. Although EUS changed the management plan for several participants, differences between groups in actual treatment and the proportion of tumours completely resected were not significant. In both groups, two thirds of initial treatment plans were for chemotherapy followed by surgery, but $40 \%$ of participants received multi-modal or palliative treatment.

Conclusion EUS has a beneficial effect on survival and generic quality of life, especially for participants initially in poorer health.

\section{P09 THE ASSOCIATION OF WEIGHT GAIN DURING ADULTHOOD WITH PROSTATE CANCER INCIDENCE AND SURVIVAL: THE NORD-TRøNDELAG HEALTH STUDY-2 COHORT, NORWAY}

doi:10.1136/jech.2010.120477.9

${ }^{1} \mathrm{C}$ Chamberlain, ${ }^{2} \mathrm{P}$ Romundstad, ${ }^{1,2} \mathrm{~L}$ Vatten, ${ }^{1} \mathrm{D}$ Gunnell, ${ }^{1} \mathrm{R}$ M Martin. ${ }^{1}$ Department of Social Medicine, University of Bristol, Bristol, UK; ${ }^{2}$ Department of Public Health, Norwegian University of Science and Technology, Trondheim, Norway

Obese men appear to have an increased risk of being diagnosed with advanced prostate cancer and of dying from the disease. Few studies have examined the impact of weight gain during adulthood on prostate cancer risk and mortality. We analysed data from 20991 Norwegian men who participated in two phases of the NordTrøndelag Health Study in 1984/1986 (HUNT-1, when aged at least 20 years) and 1995/1997 (HUNT-2). Weight and height were measured at both HUNT-1 and HUNT-2, allowing each man's change in weight and BMI during approximately 11 years of adult life to be computed. During a median of 11.3 years of follow-up after the end of HUNT-2, 649 (3\%) men developed prostate cancer. We observed an increase in prostate cancer incidence amongst men who put on weight between HUNT-1 and HUNT-2. In multivariable models, including adjustment for weight at HUNT-2, the HR for prostate cancer per one SD (6.2 kg) gain in weight was $1.16(95 \% \mathrm{CI}$ 1.03 to to 1.31 , p-trend $=0.01)$ and per one SD gain in BMI $(1.9 \mathrm{~kg} /$ $\mathrm{m}^{2}$ ) was 1.14 (95\% CI 1.00 to 1.30 , p-trend=0.04). Amongst men diagnosed with prostate cancer (any stage), there was no evidence that gain in weight prior to diagnosis was positively associated with subsequent all-cause mortality (HR per one $\mathrm{SD}$ increase in weight $=1.05 ; 95 \%$ CI 0.87 to 1.26 , p-trend $=0.63)$. The findings suggest that control of weight gain during adulthood, as well as absolute weight, has implications for prostate carcinogenesis.

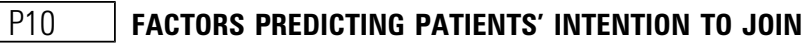 CANCER SUPPORT GROUPS AND THEIR PERCEPTIONS OF THE MOST USEFUL GROUP FORMATS}

doi:10.1136/jech.2010.120477.10

G Grande, C Missing, J Arnott. School of Nursing, Midwifery \& Social Work, University of Manchester, Manchester, UK

Background Trials have demonstrated that patients benefit from participation in cancer support groups. However, only a minority of patients join groups. Better understanding of factors predicting patients' intention to join groups and their preferred group formats is required to aid strategies to increase support group attendance and ensure that groups are suited to patients' preferences.

Objective To investigate variables predicting patients' intention to join cancer support groups and the support group formats they would find most useful.

Design Self-completed questionnaires. Factor analysis of patients' perceptions of support groups and the group formats perceived to be most useful. Comparison of patients intending and not intending to join groups and bi-variate correlation analyses of variables associated with preferred group format, using non-parametric and parametric analysis as appropriate.

Setting Recruitment through oncology outpatient clinics shortly after diagnosis.

Participant 192 patients with cancer of the colon (105), lung (57) or bladder (30). Of these, $67 \%$ were male and $33 \%$ female.

Main Measures Demographic and clinical variables, perceived social support (MSPSS), perceived control and distress over cancer (IPQ$\mathrm{R})$, strategies for coping with cancer (Brief COPE), functioning and symptoms (EORTC OLO C30), views and preferences regarding support groups (questionnaires designed from qualitative patient interviews).

Results Variables predicting participants' intention to join a group included worse family support, higher distress, coping through instrumental support seeking and little perceived difficulty in joining. Factors predicting preference for patient-led, emotionfocused groups included being female, higher distress, worse functioning in several domains, and coping through planning, positive reframing, religion, instrumental support seeking, distraction and denial. Preference for professionally led, information-based groups related to active coping and acceptance, lower education and not having a partner. Preferences for both group formats and a general intention to participate were all related to having positive views of groups and being influenced by health professionals' recommendation of groups (all findings at $\mathrm{p}<0.05$ ). More details of findings, results of multivariate analyses and data on whether patients actually joined a group will be presented at the conference.

Conclusion Patients with different characteristics prefer different support group formats. It is therefore important to tailor group formats to the needs of different groups of patients. In all instances positive perceptions of groups and recommendation from a health professionals increased perceived usefulness of groups and patients' intention to join. Both promotion of a positive image of groups and the recommendation of health professionals should therefore help increase support group attendance.

\section{Pregnancy/Early life/Birth cohorts/Health trajectories \\ P11 INCREASED RISK OF FETAL LOSS AND INFANT DEATH IN OBESE WOMEN}

doi:10.1136/jech.2010.120477.11

${ }^{1}$ P W G Tennant, ${ }^{1,2} \mathrm{~J}$ Rankin, ${ }^{1,2}{ }^{2}$ Bell. ${ }^{1}$ Institute of Health and Society, Newcastle University, Newcastle-upon-Tyne, UK; ${ }^{2}$ Regional Maternity Survey Office, Newcastleupon-Tyne, UK

Objective To investigate the association between maternal obesity and the prevalence of fetal and infant death.

Design Cohort study using prospectively collected data matched to a high-quality population-based registry data of fetal and infant death.

Setting Five maternity units in the North of England.

Participants 40932 singleton pregnancies delivered between 2003 and 2005, excluding 1092 pregnancies associated with a congenital anomaly and/or maternal pre-gestational diabetes, and 9998 pregnancies with missing maternal body mass index (BMI). 
Main Outcome Measures Prevalence, among singleton pregnancies, of late miscarriage (20-23 weeks gestation), termination of pregnancy for fetal anomaly ( $\geq 20$ weeks gestation), stillbirth ( $\geq 24$ weeks gestation), fetal death (miscarriage or antepartum stillbirth), perinatal mortality, neonatal mortality, post-neonatal mortality, and all infant mortality. Crude and adjusted (for maternal age, gestational age, where appropriate, birth weight, standardised for sex and gestational age, ethnicity, cigarette smoking status, index of multiple deprivation, and sex of infant/fetus) ORs of each outcome among maternal obese (BMI $\geq 30 \mathrm{~kg} / \mathrm{m}^{2}$ ) compared to maternal recommended BMI (BMI 18.5-24.9 kg/m²).

Results Compared to women of recommended BMI, obese women were at significantly greater risk of a fetal death (adjusted odds ratio (aOR) 2.94 (95\% CI 2.02 to 4.27), $\mathrm{p}<0.001$ ), including late miscarriage (aOR 3.27 (95\% CI 1.43 to 7.44$), \mathrm{p}=0.005$ ) and antepartum stillbirth (aOR 2.86 (95\% CI 1.88 to 4.35$), \mathrm{p}<0.001)$, perinatal death (aOR 2.54 (95\% CI 1.70 to 3.79), p<0.001), including stillbirth (aOR 2.77 (95\% CI 1.86 to 4.13$), \mathrm{p}<0.001)$ and early neonatal death (aOR 3.00 (95\% CI 1.12 to 8.03), $\mathrm{p}=0.03$ ), and infant death (aOR 2.49 ( $95 \%$ CI 1.34 to 4.62 ), $\mathrm{p}=0.004$ ), including neonatal death (aOR 2.57 (95\% CI 1.13 to 5.88), $p=0.03$ ). There was no significant association between maternal obesity and post-neonatal death (aOR 2.27 (95\% CI 0.89 to 5.80), $\mathrm{p}=0.09$ ). The effect of obesity on risk of stillbirth was greater among small-for-gestational-age fetuses, but less among current smokers. Except for higher rates of pre-eclampsia among stillbirths, no specific cause of death could explain the increased odds of fetal and infant death among the obese.

Conclusion Early-pregnancy obesity is significantly associated with fetal and infant death, independent of the known relationship with congenital anomalies. Further studies are required to investigate the specific mechanisms involved. In the meantime, women should be made aware of these risks and supported to optimise their weight before pregnancy.

\section{P12 MATERNAL SMOKING DURING PREGNANCY AND OFFSPRING BONE MASS AT AGE 9 YEARS}

doi:10.1136/jech.2010.120477.12

${ }^{1} \mathrm{C}$ Macdonald-Wallis, ${ }^{2} \mathrm{~J} H$ Tobias, ${ }^{1} \mathrm{G}$ Davey Smith, ${ }^{1} \mathrm{D}$ A Lawlor. ${ }^{1} \mathrm{M} R \mathrm{C}$ Centre for Causal Analyses in Translational Epidemiology, Department of Social Medicine, University of Bristol, Bristol, UK; ${ }^{2}$ Academic Rheumatology, Clinical Science at North Bristol, University of Bristol, Bristol, UK

Objective Studies in neonates have demonstrated an adverse relationship between maternal smoking during pregnancy and fetal bone mineral accrual. We aimed to investigate an intrauterine influence of maternal smoking in pregnancy on offspring bone mass during childhood.

Design Prospective birth cohort study: the Avon Longitudinal Study of Parents and Children (ALSPAC).

Setting Mothers were resident in Avon, UK during pregnancy and had expected delivery dates between April 1991 and December 1992. Participants and Methods Our analysis included 7121 children attending a research clinic at mean age 9.9 years. We used multiple regression models to compare associations of maternal and paternal smoking at the time of pregnancy with measures of bone size and density in the children, since a greater maternal association would provide evidence of a direct intrauterine effect on the fetus. Multivariate multiple imputations were used to account for missing data. Main Outcome Measures DXA measured total body less head $(\mathrm{TBLH})$ and spine bone mineral content (BMC), bone area $(\mathrm{BA})$ and bone mineral density (BMD).

Results Maternal smoking in any trimester of pregnancy was associated with increased TBLH BMC, BA and BMD in girls (mean difference $(95 \% \mathrm{CI}$ ) (using sex-specific SD scores): 0.13 (0.05 to
0.22 ), 0.13 (0.04 to 0.21 ) and 0.13 (0.04 to 0.22 ), respectively) but not in boys $(0.01$ ( -0.07 to 0.09$), 0.00$ ( -0.08 to 0.08$), 0.04(-0.05$ to 0.12$)$ ) in confounder-adjusted models. It was also associated with spine BMC, BA and BMD in girls (0.13 (0.03 to 0.23), 0.12 (0.03 to $0.22), 0.10(0.00$ to 0.21$))$ but not in boys $(0.03$ ( -0.06 to 0.12$), 0.00$ $(-0.09$ to 0.09$), 0.05$ ( -0.04 to 0.14$)$ ). Paternal smoking associations were similar, with no statistical evidence for a difference between maternal and paternal associations (all $p$ values $>0.15$ ). Maternal associations increased on adjustment for offspring birth weight and gestational age but attenuated to the null on adjustment for the child's height and weight at age 9 years. Further investigation indicated that these relationships were driven mainly by offspring weight rather than height.

Conclusions There was little evidence of a relationship between maternal smoking during pregnancy and bone mass in boys. Our parental comparisons and multivariable analyses suggested that the positive associations between maternal smoking and bone mass in girls were likely to be attributable to shared familial characteristics related to offspring adiposity rather than an intrauterine mechanism.

\section{P13 ASSOCIATION OF PRENATAL AND POSTNATAL SMOKING AND ALCOHOL CONSUMPTION ON BIRTH WEIGHT IN THE WHITE BRITISH POPULATION IN BRADFORD: PRELIMINARY FINDINGS FROM THE BORN IN BRADFORD STUDY}

doi:10.1136/jech.2010.120477.13

${ }^{1,2}$ E Petherick, ${ }^{2} \mathrm{R}$ Parslow, ${ }^{2} \mathrm{P}$ McKinney, ${ }^{1} \mathrm{D}$ Tufnell, ${ }^{3} \mathrm{D}$ Leon, ${ }^{1}$ Pauline Raynor, ${ }^{4} \mathrm{D}$ Lawlor, ${ }^{1} \mathrm{~J}$ Wright. ${ }^{1}$ Bradford Institute for Health Research, Bradford Royal Infirmary, Bradford, UK; ${ }^{2}$ Paediatric Epidemiology Group, University of Leeds, Leeds, UK; ${ }^{3}$ London School of Hygiene \& Tropical Medicine, London, UK; ${ }^{4}$ MRC Centre for Causal Analyses in Translational Epidemiology, Department of Social Medicine, University of Bristol, Bristol, UK

Background Exposure to cigarette smoke and heavy alcohol consumption has been known to influence birth weight and risk of being small for gestational age. Despite this knowledge, very little exploration of the timing of cigarette smoking and alcohol exposure on growth has been undertaken.

Aims To investigate the effect of dose and timing of pre-pregnancy and prenatal alcohol and cigarette exposure on birth weight.

Methods The Born in Bradford cohort includes women of diverse ethnicities, with $46 \%$ of the population being of South Asian origin. However, smoking prevalence $(5.9 \%)$ and alcohol consumption rates $(0.4 \%)$ in pregnant women of South Asian origin were too low for meaningful analyses. Therefore, analyses were restricted to the white British women who had completed a questionnaire and had a live singleton full-term birth at the Bradford Royal Infirmary, between October 2007 and August 2009. Data were available on smoking and alcohol consumption at three different time points: in the 3 months prior to pregnancy, first 3 months of pregnancy and when the baseline questionnaire was administered (26-28 weeks gestation) by trained interviewers. This information was used to classify women into three mutually exclusive categories separately for both smoking and alcohol: smoker/drinker at all time points, decreasing or quitting at different time periods throughout and nonsmokers/drinkers at all time points. Mean differences in birth weight between these categories were investigated using multivariable linear regression. The model adjusted for maternal age, baby's gender, parity, area deprivation, caffeine consumption, and mother's weight at her first antenatal clinic appointment.

Results 1933 white British mothers and their children were eligible for inclusion. $39.7 \%$ were regular smokers in the 3 months prior to pregnancy and $33.6 \%$ during pregnancy, whilst $72 \%$ consumed alcohol in the 3 months prior to pregnancy and $40 \%$ during 